\title{
Implantação das estratégias de segurança do paciente: sugestões de enfermeiros gestores
}

\section{Establishment of patient's safety strategies: suggestions of managing nurses}

\author{
Gislene Aparecida Xavier dos \\ Reis ${ }^{1, *}$ \\ Ingrid Mayara Almeida Valera' \\ Kelly Cristina Inoue" \\ Verusca Soares de Souza"I \\ Maria Antonia Ramos Costa"I \\ Laura Misue Matsuda'
}

1 Universidade Estadual de Maringá (UEM), Maringá, PR, Brasil

" Hospital Universitário de Maringá (UEM), Maringá, PR, Brasil

III Universidade Estadual do Paraná (Unespar), Paranavaí, PR, Brasil

\footnotetext{
* E-mail: gislenereis@ymail.com
}

\begin{abstract}
RESUMO
O presente estudo teve como objetivo descrever as ações que necessitam ser operacionalizadas na implantação das estratégias de segurança do paciente em hospitais, na perspectiva de enfermeiros gestores. Pesquisa descritivo-exploratória, de abordagem qualitativa, realizada com 72 enfermeiros gestores de quatro hospitais universitários públicos paranaenses. Os dados foram coletados por meio de entrevista gravada, norteada pela questão: "Fale-me sobre ações que necessitam/devem ser operacionalizadas no processo de implantação das estratégias de segurança do paciente em hospitais" e submetidos à análise de conteúdo, na modalidade temática, da qual se desvelaram as categorias: "Criação de Núcleo de Segurança integrado à Gestão da Qualidade"; "Desenvolvimento de estratégias de ensino e pesquisa a promoção da segurança do paciente" e "Ações organizacionais sistêmicas à efetividade das estratégias de segurança do paciente". Concluiu-se que as principais sugestões emitidas pelos enfermeiros foram: integração do núcleo de segurança do paciente com à assessoria de gestão da qualidade; capacitação contínua de todos os trabalhadores; parceria com o meio acadêmico e apoio maciço da alta gestão.
\end{abstract}

PALAVRAS-CHAVE: Enfermagem; Segurança do Paciente; Gestão da Segurança; Gestores de Saúde

\begin{abstract}
This study aimed to describe the actions that need to be operationalized in the implementation of patient safety strategies in hospitals in view of nurse managers. Current descriptive, exploratory and qualitative study was conducted with 72 managing nurses of four government-run university hospitals in the state of Paraná, Brazil. Data were collected by a recorded interview based on the statement: "Speak on the actions that should be implemented in the process of the establishment of patient's safety strategies in hospitals". Answers were submitted to analysis of content, theme mode, and the following categories emerged: "Establishment of a safety nucleus integrated to the quality management", "Development of teaching and research strategies for the promotion of patient's safety" and "Systemic organizational activities for the strategies of patient's safety". It may be concluded that the main suggestions of nurses were: integration of the patient's safety nucleus to quality management; continuous capacitation of all professionals; joint venture with universities and whole-hearted support by the managers.
\end{abstract}

KEYWORDS: Nursing; Patient's Safety; Perception; Safety Management; Health Manager 


\section{INTRODUÇÃO}

A segurança do paciente tem a finalidade de reduzir os riscos e danos desnecessários, relacionados ao cuidado à saúde, a um mínimo aceitável ${ }^{1}$. Há muito tempo, tal temática tem sido alvo de discussões e preocupações, pois Hipócrates, há aproximadamente 2 mil anos, ao se referir à assistência prestada ao paciente proferiu: "primeiro não causar dano"2.

Mais recentemente, em 1863, Florence Nightingale, ao perceber que morriam mais soldados por complicações patológicas evitáveis, decorrentes da assistência à saúde, do que em campos de batalha, instituiu a análise estatística para mensurar o resultado da assistência e medidas para melhorar o cuidado culminando na diminuição de óbitos preveníveis ${ }^{3}$. Porém, apenas no final da década de 1990, com a publicação do relatório To Err is Human: building a safer health system, em 1999, pelo Institute of Medicine (IOM) dos Estados Unidos da América, é que os órgãos regulatórios, gestores e profissionais da saúde voltaram a atenção para a necessidade de garantir assistência segura ${ }^{4}$.

A partir da divulgação do referido relatório, órgãos nacionais e internacionais instauraram iniciativas com o intuito de minimizar os riscos provenientes da assistência à saúde, de forma a ratificar, cada vez mais, a importância da qualidade da assistência. Nesse contexto, um estudo que analisou altas hospitalares de instituições públicas portuguesas detectou a incidência de 2,5\% de eventos adversos (EA) relacionados à assistência à saúde e, destes, 5,7\% evoluíram a óbito ${ }^{5}$. Já outra pesquisa, realizada em cinco hospitais da Itália, identificou a ocorrência de 5,2\% destes eventos, sendo que $56,7 \%$ eram evitáveis ${ }^{6}$.

A realidade brasileira não difere daqueles países, pois em estudo realizado no Estado do Rio de Janeiro, foram analisados 27.350 prontuários de pacientes, observando-se que 3,5\% dos pacientes submetidos a procedimentos cirúrgicos apresentaram EA e que a proporção de EA cirúrgicos evitáveis foi de $68,3 \%$. Além disso, esse mesmo estudo constatou que um a cada cinco pacientes sofreu danos incapacitantes, permanentes ou foram a óbito ${ }^{7}$.

A ocorrência de EA também gera elevados gastos ao sistema de saúde. Em estudo realizado em dois hospitais públicos de ensino do Estado do Rio de Janeiro, em que selecionaram de forma aleatória 622 prontuários de paciente, identificou-se que o valor médio pago pelo atendimento aos pacientes que sofreram EA foi $200,5 \%$ superior ao valor pago aos pacientes sem EA ${ }^{8}$.

Diante dos expressivos números apresentados quanto à ocorrência de $E A$, os elevados gastos com a assistência e a extrema importância de garantir cuidado seguro, órgãos nacionais e internacionais têm se empenhado em minimizar a ocorrência de danos ao paciente. Com isso, o Brasil, por meio do Programa Nacional de Segurança do Paciente (PNSP), criado em $1^{\circ}$ de abril de 2013, priorizou algumas ações com o objetivo de diminuir os riscos decorrentes da assistência à saúde a partir da implantação de estratégias para a segurança do paciente?
Dentre as estratégias estabelecidas pelo PNSP, cita-se a identificação correta do paciente; melhorar a comunicação entre os profissionais de saúde; melhorar a segurança na prescrição, no uso e na administração de medicamentos; assegurar cirurgia em local de intervenção, procedimento e pacientes corretos; higienizar as mãos para evitar infecções e reduzir o risco de quedas e úlceras por pressão?.

As estratégias referidas, em princípio, são consideradas simples e efetivas. Contudo, pela sobrecarga de trabalho, ao dimensionamento de pessoal inadequado ${ }^{10}$, da cultura organizacional negativa quanto a mudanças, gestores e órgãos hospitalares enfrentam dificuldades para a implementação dessas práticas ${ }^{11}$.

Considerando a premência de se realizar estudos que abordam a segurança do paciente, este estudo se justifica porque a literatura aponta que existe dificuldade de se implantar as estratégias de segurança do paciente em instituições hospitalares; os custos monetários e sociais, relacionados à ocorrência de EA na assistência à saúde, são elevados e os seus resultados poderão subsidiar gestores e trabalhadores no planejamento e implantação de estratégias de segurança do paciente, e, consequentemente, na prevenção de danos e agravos, oriundos do cuidado à saúde.

Ante ao exposto, esta pesquisa se pauta na questão: “Na percepção de enfermeiros gestores, quais ações devem ser operacionalizadas no processo de implantação das estratégias de segurança do paciente em hospitais?" e tem como objetivo descrever as ações que necessitam ser operacionalizadas na implantação das estratégias de segurança do paciente em hospitais, na perspectiva de enfermeiros gestores.

\section{MÉTODO}

Estudo descritivo-exploratório, de abordagem qualitativa, realizado entre janeiro e março de 2015, em quatro hospitais universitários públicos, situados no Estado do Paraná, que foram denominados de Hospital I, Hospital II, Hospital III e Hospital IV.

A saber, o Hospital I está localizado na região Leste do Estado, é de nível federal, possui 406 leitos, o quadro de enfermagem estava composto por 268 enfermeiros ${ }^{12} \mathrm{e}$, destes, 20 foram selecionados a participarem deste estudo. Nesse hospital, o processo de implantação das estratégias de segurança do paciente iniciou-se no ano de 2010.

O Hospital II, situado na região Norte do Estado, é de nível estadual, possui 313 leitos, e na equipe de enfermagem havia 116 enfermeiros $^{13}$, sendo 41 selecionados a participarem do presente estudo. Na referida instituição, a implantação das estratégias de segurança do paciente iniciou-se em 2011.

O Hospital III situa-se na região Noroeste do Estado, é de nível estadual, possui 123 leitos, 89 enfermeiros ${ }^{14} \mathrm{e}$, destes, 18 foram selecionados para participarem do presente estudo. Em 2014, iniciou-se o processo de implantação das estratégias de segurança do paciente. 
Por fim, o Hospital IV, localizado na região Oeste do Estado, é também de nível estadual, possui 195 leitos e 89 enfermeiros ${ }^{15}$, sendo 15 eleitos a participarem desta pesquisa. Em 2014, as estratégias de segurança do paciente começaram a serem implantadas.

A coleta de dados procedeu-se, primeiramente, pela autorização formal dos quatro hospitais. Em seguida, os dados pessoais dos enfermeiros (nome, cargo, data de admissão, contato telefônico e/ou endereço eletrônico) foram obtidos com o auxílio do setor de recursos humanos. Após, foram selecionados todos os enfermeiros com cargo de gestão, que atuavam há mais de um ano na instituição, que ocupavam o cargo atual há pelo menos seis meses. Foram excluídos os indivíduos que se negaram a participar da pesquisa ou que estavam em serviço, mas que por algum motivo não puderam participar do estudo, e os que estavam de atestado médico ou férias.

Posteriormente, realizou-se contato (telefônico, e/ou por correio eletrônico, e/ou pessoal) com os enfermeiros para o fornecimento de informações referentes ao objetivo do estudo, a técnica de coleta de dados e as implicações éticas. Mediante o aceite informal, a entrevista foi agendada de acordo com a disponibilidade de cada participante. Previamente à realização das entrevistas, que foi realizada em local reservado, foi disponibilizado, em duas vias o Termo de Consentimento Livre e Esclarecido (TCLE) para leitura e assinatura.

Cumpre ressaltar que, para informar e convidar os participantes quanto à realização do estudo, foram estabelecidas as seguintes estratégias: três tentativas de contatos telefônicos em horários e em dias alternados. Caso a pesquisadora não obtivesse resposta, eram encaminhados três e-mails, com intervalo de sete dias. Mediante o não recebimento de resposta, o contato pessoal no ambiente de trabalho foi a última alternativa viabilizada.

No que tange à coleta de dados propriamente dita, ela ocorreu por meio da aplicação de um formulário com questões objetivas, relacionadas à caracterização sociodemográfica e laboral dos participantes e, na sequência, foi realizada entrevista gravada, apoiada na questão norteadora: "Fale-me sobre ações que necessitam/devem ser operacionalizadas no processo de implantação das estratégias de segurança do paciente em hospitais".

As entrevistas foram transcritas na íntegra e, após, foram submetidas à análise de conteúdo, na modalidade temática ${ }^{16}$, respeitando-se a fase de pré-análise, de exploração do material e do tratamento dos dados. A referida técnica é descrita como a utilização de ferramentas analíticas que possibilitam revelar o significado do conteúdo da mensagem emitida pelos participantes do estudo, por intermédio de deduções provenientes de procedimentos sistematizados.

$\mathrm{Na}$ apresentação dos resultados, os excertos/trechos/verbatins dos relatos foram editados no sentido de corrigir possíveis erros gramaticais sem, no entanto, alterar o conteúdo dos mesmos. Além disso, em algumas partes, foram acrescidos alguns termos/palavras entre colchetes. Para preservar a identificação dos participantes, os excertos foram codificados pela letra "E", a qual significa "Entrevista", e "H", para denominar o "Hospital" onde foi realizada a entrevista, além de um número arábico indicando a sequência em que as mesmas foram realizadas.

Este estudo, que faz parte de um projeto maior, intitulado "Gestão em Saúde/Enfermagem: Qualidade e Segurança do Paciente em Hospitais Universitários", foi submetido ao Comitê de Ética em Pesquisa Envolvendo Seres Humanos, da Universidade Estadual de Maringá (UEM) e recebeu parecer favorável sob número 1.158.794.

\section{RESULTADOS E DISCUSSÃO}

Nos quatro hospitais, de acordo com os critérios de inclusão e exclusão, foram selecionados 94 enfermeiros gestores, sendo 20 (21,0\%) do Hospital I, 41 (44,0\%) do Hospital II, 18 (19,0\%) do Hospital III e 15 (16,0\%) do Hospital IV.

Do total de enfermeiros gestores selecionado, $72(77,0 \%)$ aceitaram participar da pesquisa, dos quais $26,4 \%$ trabalhavam no $\mathrm{H} 1 ; 39,0 \%$ no $\mathrm{H} 2 ; 15,2 \%$ no $\mathrm{H} 3$ e $19,4 \%$ no $\mathrm{H} 4$. Em todos os hospitais houve maior prevalência do sexo feminino $(\mathrm{H} 1=94,7 \%$; $\mathrm{H} 2=92,9 \%, \mathrm{H} 3=82,0 \%, \mathrm{H} 4=86,0 \%$ ). Com relação à faixa etária, $47,3 \%$ dos enfermeiros gestores do $\mathrm{H} 1$ tinham entre 50 e 59 anos; $42,8 \%$ do $\mathrm{H} 2$ e $63,7 \%$ do $\mathrm{H} 3$ tinham entre 40 e 49 anos, já 71,4\% dos participantes do $\mathrm{H} 4$ tinham entre 30 e 39 anos.

Da análise dos dados emergiram três unidades temáticas: Criação de núcleo de segurança integrado à gestão da qualidade; Desenvolvimento de estratégias de ensino e pesquisa à promoção da segurança do paciente; e Ações organizacionais sistêmicas à efetividade das estratégias de segurança do paciente.

Criação de núcleo de segurança integrado à assessoria de gestão da qualidade

Para que os hospitais criem um ambiente seguro é necessário estabelecer parcerias entre os serviços da instituição, no sentido de dedicarem-se ao desenvolvimento das estratégias de segurança do paciente ${ }^{17}$.

Em consonância com a afirmação anterior, no que tange às ações para a implantação das estratégias de segurança do paciente, os enfermeiros gestores apontaram: "[...] é necessário criar uma assessoria de gestão da qualidade junto ao núcleo de segurança [do paciente], com profissionais dedicados exclusivamente aos trabalhos relacionados à qualidade e à segurança" (H1E1); "[...] é importante formar um serviço de qualidade e um núcleo de risco para que cada profissional se responsabilize pela implantação de um protocolo" (H1E11).

Os excertos H1E1 e H1E11 sugerem o trabalho conjunto entre o Núcleo de Segurança do Paciente (NSP) e a assessoria de qualidade. Talvez isso ocorra porque, no hospital investigado, ambos os serviços atuam de forma integrada e subsidiam todas as ações voltadas para o cuidado seguro, ratificando então, a necessidade de criá-los em outras instituições. 
Segundo a Agência Nacional de Vigilância Sanitária (Anvisa), as atividades desenvolvidas pelo NSP e pela assessoria de qualidade devem estar alinhadas para garantir assistência segura ao paciente. Neste sentido, o objetivo do NSP constitui-se em promover e apoiar a implantação de estratégias de segurança do paciente ${ }^{18}$, atuando assim, como articulador e incentivador das diversas unidades do hospital que gerenciam riscos e promovem ações de qualidade em serviços de saúde. Já à assessoria da qualidade cabe prevenir, detectar e solucionar problemas relacionados com a qualidade e a segurança do paciente ${ }^{18}$, visto esta representar uma dimensão da qualidade da assistência à saúde.

Os relatos H1E1 e H1E11 também apontam a necessidade dos profissionais atuarem exclusivamente no NSP ou no serviço de gestão da qualidade. Contudo, tal necessidade configura-se, em algumas instituições, em um desafio para os gestores, por conta do dimensionamento inadequado de enfermeiros. Em estudo realizado em um hospital geral, os enfermeiros investigados relataram a dificuldade em integrar as atividades assistenciais às gerenciais, suscitando a necessidade de ter um profissional para cada função, com vista à assistência integral ao paciente, à melhor organização do processo de trabalho e, por conseguinte, à minimização de riscos na assistência ${ }^{19}$.

Cumpre ressaltar que, no discurso de H1E11, é pontuada a necessidade de cada profissional se responsabilizar pelo gerenciamento de um protocolo. Essa percepção contradiz a Resolução da Diretoria Colegiada (RDC) da Anvisa $n^{\circ} 36$, de 25 de julho de 2013, a qual recomenda que uma equipe multiprofissional, e não apenas um profissional, participe do processo de desenvolvimento das estratégias de segurança do paciente ${ }^{20}$. Corrobora a tal afirmativa pesquisa que aponta a importância da integração entre diferentes categorias profissionais para a efetivação da implantação das estratégias de segurança do paciente ${ }^{21}$.

Para que o serviço de gestão da qualidade alcance seus objetivos também é importante que esteja em consonância com a alta gestão do hospital, conforme consta no seguinte relato: “[...] é importante ter um Serviço de Qualidade diretamente ligado ao Diretor e que esse gestor, aceite e incorpore essas ideias [estratégias de segurança do paciente]" (H1E2).

O envolvimento da alta gestão é basilar à implantação de estratégias seguras para o paciente, para os profissionais, e também para a própria instituição. $O$ apoio da cúpula administrativa é essencial para a promoção do cuidado seguro porque pode proporcionar aos profissionais o sentimento de que não estão sozinhos nessa missão $0^{22}$. Fato este destacado pelas organizações mundiais através do estímulo ao estabelecimento de uma cultura de segurança nas instituições, pautada em uma forma não punitiva de enfrentamento ao erro e que deve envolver todos os trabalhadores dos mais diferentes níveis de formação?.

O apoio da alta gestão da instituição nos processos de segurança do paciente não deve ser interpretado como novidade ou benevolência porque, de acordo com os preceitos da gestão da qualidade $^{2}$, a alta gestão, por ter poder de decisão e de captar recursos, deve compor a primeira linha nos processos que envolvem mudanças estratégicas no gerenciamento.

Considera-se, portanto, que a integração entre o NSP e a Assessoria de Gestão da Qualidade e o apoio da alta gestão, são quesitos de suma importância para a efetivação das práticas seguras ao paciente.

Desenvolvimento de estratégias de ensino e pesquisa à promoção da segurança do paciente

Promover a educação profissional configura-se em momento singular à promoção da assistência segura. "Acredito que a primeira etapa é fazer a sensibilização, para que todas as pessoas que estarão envolvidas entendam o que é, o porquê e o quanto que isso irá trazer de benefícios para o paciente e para o profissional" (H1E17); "Primeiramente tem que esclarecer para todos os colaboradores o que é a segurança [do paciente] e o que é o Programa [Programa Nacional de Segurança do Paciente]" (H4E14).

De acordo com os excertos, os entrevistados entendem a importância da realização de educação em serviço, no processo de implantação das medidas de segurança. Isso porque, paralelamente à integração do NSP com a Assessoria de Gestão da Qualidade, é elementar que os colaboradores conheçam a temática e atuem no sentido de garantir o cuidado seguro, não só ao paciente, mas também ao trabalhador, de forma sistematizada, contextualizada e contínua.

Destaca-se que os benefícios advindos da prática da assistência segura ao colaborador se pautam no reconhecimento de que uma vez que os erros são diagnosticados, as falhas no processo podem ser retificadas, proporcionando satisfação profissional por desempenhar as atividades laborais em ambiente seguro ${ }^{23}$.

Os riscos de o paciente sofrer EA são elevados nas organizações hospitalares que não privilegiam o aprendizado; há escassez de informações ou quando estas são transmitidas de forma distor$\mathrm{cida}^{24}$. Nesse aspecto, a educação em serviço constitui-se ferramenta eficaz para subsidiar as mudanças no processo assistencial porque o aprimoramento de conhecimentos contribui para o desenvolvimento de habilidades e competências necessárias à prática segura ${ }^{25}$.

Para disseminar o conhecimento em torno da segurança do paciente, os enfermeiros sugeriram: “[...] acho que, principalmente, envolver discussões em grupos pequenos, escutando realmente, sanando dúvidas [...]" (H1E17); "Sugiro que os hospitais realizem capacitações in loco, que [o profissional responsável pela capacitação] vá até a unidade e realize o treinamento com um ou dois colaboradores" (H2E4).

A realização de capacitações em pequenos grupos, indicados por H1E17 e H2E4 é outra sugestão importante porque, de acordo com os participantes do estudo, em treinamentos realizados com número maior de pessoas, apenas algumas eram sensibilizadas. Estudo de Barbato, Corrêa e Souza ${ }^{26}$ corrobora tal afirmativa, referindo que capacitações realizadas em pequenos grupos proporcionam melhor aprendizado porque o colaborador pode sanar 
dúvidas, interagir melhor entre os membros do grupo e com isso trocar experiências e conhecimentos.

Além da realização de capacitações em pequenos grupos, houve sugestão de que é necessário desenvolvê-las de forma contínua e sistematizada.

[...] precisa ter uma educação muito forte [bem planejada] no sentido de estar sempre desenvolvendo campanhas, criando meios para que essas estratégias sejam fortalecidas e realmente promovam mudanças na cultura [organizacional]. (H1E15)

Tem que estar sempre atualizadas [às capacitações] para informar os profissionais diariamente quanto às resoluções, portarias do Ministério da Saúde [...]. Não é porque as estratégias foram implantadas que não precisamos de cursos. Pelo contrário senão, as pessoas deixam de dar importância [para as estratégias de segurança do paciente]. (H1E2)

Os depoimentos mencionados apontam a necessidade de os momentos de ensino/educação serem realizados com periodicidade, com intuito de fortalecer o conhecimento prévio, mas também adquirir novas informações e práticas. Na perspectiva da segurança do paciente, Yoshikawa et al. ${ }^{27}$ ilustram que, além da realização de capacitações, é necessário que estas sejam realizadas constantemente, porque a periodicidade das ações educativas é importante para se prevenir falhas assistenciais.

O envolvimento do meio acadêmico, também coaduna para a garantia da assistência segura, como exposto a seguir:

Sugiro que os hospitais realizem a integração docente assistencial, porque é importante a parceria com o Departamento de Enfermagem [...], os professores junto com os alunos podem auxiliar os gestores nas atividades para a implantação das estratégias [de segurança do paciente]. (H2E11)

[...] é importante ter um docente que participa efetivamente, que dê suporte, que traga novos conhecimentos, que promova discussão com os profissionais. (H3E8)

A parceria entre os serviços de saúde com as instituições de ensino é elementar para a troca de conhecimentos e para a implantação de diferentes estratégias. Isso porque essa relação é considerada indissociável visto que a instituição de ensino proporciona a formação/aquisição de conhecimentos e a transformação da realidade ${ }^{28}$. Por sua vez, a instituição de saúde possibilita a inserção do discente/estudante no contexto da prática assistencial e ao desenvolvimento de ações em prol da segurança do paciente ${ }^{28}$.

Talvez em decorrência da recente normativa brasileira quanto à implantação das estratégias de segurança, os enfermeiros gestores elencaram a necessidade de conhecer instituições hospitalares em que a implantação das estratégias esteja avançada. "Conhecer serviços que já implantaram as estratégias, para verificar como realizaram e se as ações podem ser replicadas" (H1E15); "Procurar outros serviços de saúde que já trabalham com a segurança do paciente para conhecer como foi o processo de implantação" (H2E1); "Sugiro que realizem visitas a outros hospitais que já implantaram ou que estão implantando as estratégias [de segurança do paciente], para conhecer como foi a construção, quais foram as barreiras enfrentadas [...]" (H4E13).

Apesar da existência da normativa brasileira que orienta quanto à implantação do cuidado seguro, são necessárias visitas a outras organizações, com o intuito de conhecer outras realidades em que a prática da assistência segura não seja novidade. Essa prática, no mundo corporativo, denomina-se Benchmarking e consiste na avaliação das organizações quanto às melhores práticas gerenciais e/ou, organizacionais, com o objetivo de identificar oportunidades de melhoria ${ }^{29}$. Mediante isso, ao conhecer outras realidades hospitalares, os enfermeiros gestores poderão realizar intervenções mais assertivas quanto à implantação das estratégias almejadas.

Nesta categoria, constata-se que os enfermeiros percebem a importância de a instituição realizar ações com diferentes escopos, com o intuito de agregar conhecimento que contribui ao processo de implantação das estratégias de segurança do paciente.

Ações organizacionais sistêmicas à efetividade das estratégias de segurança do paciente

Os enfermeiros gestores sugerem que há necessidade de todos os colaboradores da organização estarem envolvidos, de forma efetiva, com a segurança do paciente:

[...] desde os seguranças, técnicos que consertam equipamentos até a alta gestão precisam falar a mesma língua [...]. (H1E5)

[...] tem que ter o envolvimento, a sensibilização de toda parte administrativa e assistencial. Caso contrário não se consegue caminhar. (H1E17)

[...] é necessário que haja sintonia entre as direções, os líderes dentro da instituição. Todos têm que estar em sintonia, tem que querer implantar e fazer a cultura de segurança ser uma verdade dentro da instituição para depois, implantar as estratégias [de segurança do paciente]. (H4E7)

O envolvimento e a sintonia entre todos os profissionais da organização, sugeridos pelos enfermeiros, coaduna com a literatura $^{30}$ e vai ao encontro da recomendação de se estabelecer uma cultura de segurança nas instituições, quando ressalta que a participação ativa de todas as categorias profissionais é importante para o sucesso da implantação de práticas seguras porque o trabalho integrado entre os colaboradores proporciona maior eficiência na solução de problemas e ao desenvolvimento de estratégias eficazes, com vista à assistência segura ${ }^{18}$. 
Diante da necessidade da implantação das estratégias de segurança do paciente, é necessário que a alta gestão do hospital coloque esse assunto em pauta e exija de seus liderados o cumprimento de ações seguras, conforme conta nos seguintes trechos: "As estratégias devem ser implantadas independente da vontade dos sujeitos, tem que ser algo que a Direção acredite ser importante e que estabeleça como diretriz da instituição, para ser alcançado" (H3E2); “A Diretoria deve incentivar a implantação das estratégias de segurança do paciente porque [as estratégias] perpassam pelo recurso financeiro [...]" (H3E6).

O incentivo da alta gestão à implantação das estratégias de segurança do paciente é importante para que os profissionais percebam que a alta cúpula administrativa reconhece a importância e apoia o desenvolvimento de práticas seguras. Nessa ótica, são crescentes as iniciativas para a assistência segura com o envolvimento de todos os profissionais da instituição, com destaque para o direcionamento da alta gestão ${ }^{31}$. Ademais, o apoio da alta administração à implantação de práticas seguras faz-se necessário porque fornece subsídios financeiros, recursos humanos e determinam o delineamento dos processos institucionais para que os profissionais atuem ao cuidado seguro.

Nesta categoria, evidenciou-se que as ações organizacionais devem ser realizadas de forma sistêmica, compreendendo o envolvimento de todos os profissionais da instituição e também o apoio da alta gestão.

\section{CONCLUSÕES}

Nesta investigação, obtiveram-se sugestões de enfermeiros gestores à implantação das estratégias de segurança do paciente. Dentre os apontamentos, constam: integração entre o NSP e a Assessoria de Gestão da Qualidade, envolvimento dos serviços de saúde junto às instituições de ensino, apoio da alta administração e educação em serviço.

Como principal limitação deste estudo, aponta-se o fato de as instituições investigadas ainda se encontrarem em fases (distintas) de implantação das estratégias de segurança do paciente, todos os hospitais serem de ensino e a participação exclusiva de gestores. Apesar disso, considera-se que os seus resultados fornecem subsídios para a tomada de decisão de gestores que almejam a efetivação de tais estratégias.

Cabe aludir, por fim, que novos estudos relacionados com a segurança do paciente são necessários, principalmente aqueles voltados à constatação do impacto das ações educacionais para a promoção do cuidado seguro.

\section{REFERÊNCIAS}

1. World Health Organization. Conceptual framework for the international classification for patient safety: version 1.1: final technical report. Geneva: World Health Organization; 2009.

2. Wachter RM. Compreendendo a segurança do paciente. 2a ed. Porto Alegre: AMGH; 2013.

3. Tronchin DMR, Melleiro MM, Tokahashi RT. A qualidade e a avaliação dos serviços de saúde e de enfermagem. In: Kurcgant P, coordenadora. Gerenciamento em enfermagem. Rio de Janeiro: Guanabara Koogan; 2010. p. 71-84.

4. Kohn LT, Corrigan JM, Donaldson MS, editors. To err is human: building a safer health system. Washington, DC: Institute of Medicine/National Academy Press, 2000.

5. Mansoa A, Vieira C P, Ferrinho P, Nogueira P, Varandas L. Eventos adversos na prestação de cuidados hospitalares em Portugal no ano de 2008. Rev Port Salud Pub. 2011;29(2):116-22. doi:10.1016/S0870-9025(11)70015-3

6. Tartaglia R, Albolino S, Bellandi T, Bianchini E, Biggeri A, Fabbro $G$ et al. Eventi avversi e conseguenze prevenibili: studio retrospettivo in cinque grandi ospedali italiani. Epidemiol Prev. 2012;36(3/4):151-61.

7. Moura MLO, Mendes W. Avaliação de eventos adversos cirúrgicos em hospitais do Rio de Janeiro. Rev Bras Epidemiol. 2012;15(3): 523-35. doi:10.1590/S1415-790X2012000300007

8. Porto S, Martins M, Mendes W, Travassos C. A magnitude financeira dos eventos adversos em hospitais no Brasil. Rev Port Saúde Pública. 2010; Vol Temat(10):74-80.
9. Ministério da Saúde (BR). Portaria $n^{\circ} 529$, de $1^{\circ}$ de abril de 2013. Institui o Programa Nacional de Segurança do Paciente (PNSP). Diário Oficial União. 2 abr 2013.

10. Inoue KC, Matsuda LM. Segurança do paciente: abordando um antigo problema. Cienc Cuid Saude. 2013;12(2):208-9. doi:10.4025/cienccuidsaude.v12i2.23880

11. Santana HT, Siqueira HN, Costa MMM, Oliveira DCAN, Gomes $S M$, Sousa FC et al. A segurança do paciente cirúrgico na perspectiva da vigilância sanitária: uma reflexão teórica. Vig Sanit Debate. 2014;2(2):34-42. doi:10.3395/vd.v2i2.124

12. Universidade Federal do Paraná. Hospital de Clínicas. HC em números. Curitiba; 2015[acesso 2 dez 2015]. Disponível em: http://www.hc.ufpr.br/?q=node/83

13. Universidade Estadual de Londrina. Hospital Universitário Regional do Norte do Paraná. Hospital Universitário. Londrina; 2015[acesso 20 jun 2015]. Disponível em: http://www.uel.br/hu/portal/

14. Universidade Estadual de Maringá. Hospital Universitário de Maringá. Estrutura. Maringá; 2015[acesso 20 jun 2015]. Disponível em: http: / /www.hum.uem.br/estrutura/

15. Universidade Estadual do Oeste do Paraná. Hospital Universitário do Oeste do Paraná.Dados estatísticos. Cascavel; 2015[acesso 20 jun 2015]. Disponível em: http://www5. unioeste.br/portal/huop/institucional/dados-estatisticos-huop

16. Bardin L. Análise de conteúdo. Lisboa; Edições 70; 2011.

17. World Health Organization. WHO patient safety. Geneva: World Health Organization; 2013[acesso 23 out. 2015]. Disponível em: http://www.who.int/topics/patient_safety/en 
18. Agência Nacional de Vigilância Sanitária - Anvisa. Assistência segura: uma reflexão teórica aplicada à prática. Brasília, DF: Agência Nacional de Vigilância Sanitária; 2013.

19. Giordani JN, Bisogno SBC, Silva LAA. Percepção dos enfermeiros frente às atividades gerenciais na assistência ao usuário. Acta Paul Enferm. 2012;25(4):511- 6. doi:10.1590/S0103-21002012000400005

20. Agência Nacional de Vigilância Sanitária - Anvisa. Resolução - RDC n 36, de 25 de julho de 2013. Institui ações para a segurança do paciente em serviços de saúde e dá outras providências. Diário Oficial União. 20 nov 2013;Seção 1:77.

21. Marques LFG, Romano-Lieber N. S. Segurança do paciente no uso de medicamentos após a alta hospitalar: estudo exploratório. Saude Soc. 2014;23(4):1431-44. doi:10.1590/S0104-12902014000400025

22. Mello JF, Barbosa SFF. Cultura de segurança do paciente em terapia intensiva: recomendações da enfermagem. Texto Contexto Enferm. 2013;22(4):1124-33. doi:10.1590/S0104-07072013000400031

23. Reis CT. A cultura de segurança do paciente: validação de um instrumento de mensuração para o contexto hospitalar brasileiro [tese]. Rio de Janeiro: Escola Nacional de Saúde Pública Sergio Arouca, Fundação Oswaldo Cruz; 2013.

24. Manzo BF, Brito MJM, Alves M. Influência da comunicação no processo de acreditação hospitalar. Rev Bras Enferm. 2013;66(1):46-51. doi:10.1590/S0034-71672013000100007

25. Queiroz ACS, Albuquerque LG, Malik AM. Gestão estratégica de pessoas e inovação:estudos de caso no contexto hospitalar. Rev Adm. 2013;48(4):658-70. doi: $10.5700 /$ rausp 1112

26. Barbato RG, Corrêa AK, Souza MCBM. Aprender em grupo: experiência de estudantes de enfermagem e implicações para a formação profissional. Esc Anna Nery. 2010;14(1):48-55. doi:10.1590/S1414-81452010000100008

27. Yoshikawa JM, Sousa BES, Peterlini MAS, Kusahara DM, Pedreira MLG, Avelar AFM. Compreensão de alunos de cursos de graduação em enfermagem e medicina sobre segurança do paciente. Acta Paul Enferm. 2013; 26(1):21-9. doi:10.1590/S0103-21002013000100005

28. Marin MJS, Oliveira MAC, Otani MAP, Cardoso CP, Moravcik MYAD, Conterno LO et al. A integração ensino-serviço na formação de enfermeiros e médicos: a experiência da FAMEMA. Ciênc Saúde Coletiva. 2014;19(3):967-74. doi:10.1590/1413-81232014193.09862012

29. Moriarty J, Smallman C. En route to a theory of benchmarking. Benchmarking: Int J. 2009;16(4):484-503. doi:10.1108/14635770910972423

30. Araújo MPS, Oliveira AC. Contribuições do programa "cirurgias seguras salvam vidas" na assistência ao paciente cirúrgico: revisão integrativa. Rev Enferm UFPE on line. 2015;9(4):7448-57. doi:10.5205/reuol.7275-62744-1-SM.0904201533

31. Oliveira RM, Leitão IMTA, Silva LMS, Figueiredo SV, Sampaio RL, Gondim MM. Estratégias para promover segurança do paciente: da identificação dos riscos às práticas baseadas em evidências. Esc Anna Nery. 2014;18(1):122-9. doi:10.5935/1414-8145.20140018

Conflito de Interesse

Os autores informam não haver qualquer potencial conflito de interesse com pares e instituições, políticos ou financeiros deste estudo.

Esta publicação está sob a licença Creative Commons Atribuição 3.0 não Adaptada.

Para ver uma cópia desta licença, visite http://creativecommons.org/licenses/by/3.0/deed.pt_BR. 